\title{
Comportamiento geoquímico del molibdeno y sus isótopos en el ambiente sedimentario - Un resumen bibliográfico
}

\author{
Geochemical behavior of molybdenum and its isotopes in \\ the sedimentary environment - A bibliographic review
Jean Carlos Montero-Serrano ${ }^{1,2}$, Manuel Martínez-Santana², Nicolas Tribovillard', Armelle Riboulleau ${ }^{1}$ y Grony Garbán ${ }^{2}$

\author{
${ }^{1}$ Université Lille 1, Laboratoire Géosystèmes, UMR 8157 - CNRS, bâtiment SN5, 59655 Villeneuve d'Ascq cedex, France \\ ${ }^{2}$ Instituto de Ciencias de la Tierra, Universidad Central de Venezuela, Caracas 1010-A, Apartado Postal 3895, Venezuela \\ jeanmontero@yahoo.es
}

\begin{abstract}
Numerous studies have been devoted to trace metals and their value in assessing the paleoredox conditions of ancient depositional settings. Among them, molybdenum (Mo) is frequently cited as an effective paleoredox proxy for sediments and sedimentary rocks. Different mechanisms that imply Mo removal from the sea and its later concentration in sediments are widely discussed in the literature. Due to the capacity to form bond of the covalent type and its atomic properties, the dissolved $\mathrm{Mo}$ in the ocean $\left(\mathrm{MoO}_{4}^{2-}\right)$, under anoxic-euxinic (rich in $\mathrm{H}_{2} \mathrm{~S}$ ) conditions, reacts to form thiomolybdates that subsequently are scavenged by forming bonds with sulfur-rich organic molecules, Fe-Mn oxides (ferromanganese nodules) and iron sulfide (pyrite); this adsorption drives an isotope fractionation of Mo that is marked in $\delta^{9895} \mathrm{Mo}$, with an average of $-0.7 \%$ in oxic conditions and with an average of $2.3 \%$ in anoxic-euxinic conditions. The use of Mo concentration as a marker of paleoredox conditions needs to be complemented with other independent markers of redox conditions (e.g. U, V, TOC, Re, Os, Fe). Mo isotopic composition seems to be a promising proxy to reconstruct and detect changes in the paleoredox status of the marine sedimentary environments.
\end{abstract}

Key words: Geochemistry, Molybdenum isotopes, paleoredox conditions, paleoenvironments

\section{Introducción}

La concentración de oxígeno en los océanos está íntimamente relacionada con el ciclo biogeoquímico del carbono. Por ello, en general los biogeoquímicos y geocientíficos se interesan en reconstruir y documentar los cambios de las condiciones reductoras en el océano a través del tiempo geológico. Debido a los avances analíticos instrumentales recientes, se han podido identificar nuevos marcadores paleoredox (e.g. isótopos de molibdeno, Re, Os), y mejorar los ya existentes (e.g. Mo-COT, Re/Mo), catalizando de esta manera un
Resumen.- Son numerosas las investigaciones dedicadas al estudio de metales traza y sus aplicaciones como indicadores de procesos geoquímicos en ambientes sedimentarios antiguos. Entre estos metales, el molibdeno (Mo) es frecuentemente citado como un marcador efectivo de condiciones redox. En la literatura están propuestos diversos mecanismos que permiten explicar la movilización de Mo en los océanos y su posterior concentración en los sedimentos. Debido a la capacidad de formar enlaces de tipo covalente y a sus propiedades atómicas, el Mo disuelto en los océanos $\left(\mathrm{MoO}_{4}^{2-}\right)$, bajo condiciones anóxicas-euxínicas (ricas en $\mathrm{H}_{2} \mathrm{~S}$ ), reacciona para formar tiomolibdatos que son posteriormente adsorbidos por moléculas orgánicas ricas en sulfuros, óxidos de Fe-Mn (nódulos de hierromanganeso) y por sulfuros de hierro (pirita). Esta absorción produce un fraccionamiento en los isótopos de Mo, que es marcada en los $\delta^{98 / 95}$ Mo con un promedio de $-0,7 \%$ en condiciones óxicas y un promedio de $2,3 \%$ en condiciones anóxicas-euxínicas. El uso de la concentración de Mo como marcador de condiciones paleoredox necesita ser complementado con otros marcadores independientes de condiciones redox (e.g. U, V, TOC, Re, Os, Fe). La composición isotópica del Mo parece ser un poderoso marcador para reconstruir y detectar cambios en las condiciones de paleoxigenación en ambientes sedimentarios marinos.

Palabras clave: Geoquímica, isótopos de Molibdeno, condiciones de paleoxigenación, paleoambiente

renacimiento de las investigaciones en la geoquímica redox de los elementos. En este sentido, el molibdeno sedimentario es comúnmente utilizado en la literatura como un marcador de condiciones paleoredox. Un enriquecimiento de este elemento en sedimentos y rocas sedimentarias, determinado mediante el factor de enriquecimiento (FE): $\mathrm{FE}_{\mathrm{Mo}}=\mathrm{Mo} / \mathrm{Al}_{\text {muestra }}: \mathrm{Mo} / \mathrm{Al}_{\text {lutita promedio }}$ (ver discusión en Tribovillard et al. 2006), es interpretado como el reflejo de bajos potenciales redox en el ambiente sedimentario (Sageman et al. 2003, Tribovillard et al. 2006, Pearce et al. 2008, Böning et al. 2009). De esta manera, las facies anóxicas estarán generalmente 
enriquecidas en molibdeno con respecto a las facies óxicas y subóxicas. Los mecanismos de este proceso de enriquecimiento han sido estudiados en diversos trabajos (e.g. Emerson \& Huested 1991, Crusius et al. 1996, Helz et al. 1996, Zheng et al. 2000). El enriquecimiento y movilización del molibdeno en el ambiente sedimentario marino también produce un fraccionamiento en los isótopos de este metal (Siebert et al. 2003, Arnold et al. 2004, Poulson et al. 2006, Pearce et al. 2008, Neubert et al. 2008, Archer \& Vance 2008). De esta manera, cambios en las condiciones redox en el ambiente de depositación marina pueden ser determinados a través de la composición isotópica del molibdeno (e.g. $\delta^{98 / 95} \mathrm{Mo}_{\text {óxico }}$ $\sim-0,7 \%$ o, $\delta^{98 / 95} \mathrm{Mo}_{\text {subóxico }} \sim 1,6 \%, \delta^{98 / 95} \mathrm{Mo}_{\text {euxínico }} \sim 2,3 \%$ ).

Estudios recientes (e.g. Siebert et al. 2003, Poulson et al. 2006, Neubert et al. 2008) destacan la necesidad de mejorar la comprensión de los mecanismos de movilización del molibdeno bajo condiciones reductoras en diferentes ambientes de sedimentación y escalas de tiempo geológico. En este sentido, presentamos un resumen bibliográfico sobre el comportamiento geoquímico del molibdeno y sus isótopos en el ambiente sedimentario, haciendo énfasis en los mecanismos de incorporación y preservación de este metal en ambientes marinos, con el objetivo de proponer un modelo esquemático y conceptual del comportamiento geoquímico de este elemento en el ambiente sedimentario marino, así como también, de generar el conocimiento necesario que permita su interpretación en futuros estudios de condiciones de paleoxigenación.

\section{Consideraciones generales del molibdeno}

El molibdeno (Mo) elemental es un metal de la segunda serie de transición que se encuentra junto al cromo $(\mathrm{Cr}) \mathrm{y}$ tungsteno (W), en el bloque 'd' y grupo 6 de la tabla periódica; de color gris metálico, que se presenta en la naturaleza con un estado de oxidación +6 ; su electronegatividad de Pauling de 2,16 le permite formar enlaces de carácter covalente. Un resumen de sus propiedades atómicas y físicas se presenta en la Tabla 1. El Mo presenta una relación diagonal con el vanadio (V) y el renio $(\mathrm{Re})$ en la tabla periódica, un ejemplo de ello, es que en sus estados de oxidación más altos, estos elementos forman oxoaniones tetraédricos $\mathrm{VO}_{4}{ }^{3-}$, $\mathrm{MoO}_{4}^{2-}, \mathrm{y} \mathrm{ReO}_{4}^{-}$; el renio puede encontrarse asociado a minerales de disulfuro de molibdeno (molibdenita, $\mathrm{MoS}_{2}$ ). Por ende, es común encontrar en la literatura trabajos donde se relacionen estos elementos como indicadores de procesos redox (Crusius et al. 1996, Morford \& Emerson 1999, Pearce et al. 2008).
Geoquímicamente, el Mo se encuentra principalmente en la naturaleza en forma de molibdato $\left(\mathrm{MoO}_{4}{ }^{2-}\right)$. Puede establecer puentes de oxígeno, formando polianiones que están unidos por ligandos Mo-O-Mo. El Mo es poco reactivo en soluciones acuosas oxigenadas, y por lo tanto es un elemento conservativo en los océanos; tiempo de residencia de $\sim 800$ ka (Morford \& Emerson 1999). El Mo es tan poco reactivo en el agua de mar que es el metal de transición más abundante en los océanos $(\sim 105 \mathrm{nmol}$ $\mathrm{kg}^{-1}$ ), a pesar de ser un componente cuya concentración en la corteza se encuentra en partes por millón (ppm). Por el contrario, el Mo es fácilmente removido de la columna de agua en ambientes anóxicos - euxínicos, con altas concentraciones de $\mathrm{H}_{2} \mathrm{~S}$, de modo que el enriquecimiento de Mo en sedimentos es considerado diagnóstico de condiciones de depositación reductoras (Calvert \& Pedersen 1993, Anbar 2004, McManus et al. 2005, Tribovillard et al. 2006).

Debido a la capacidad de formar enlaces de tipo covalente y a sus propiedades atómicas (Tabla 1), el Mo es el principal metal de transición que guarda una relación estrecha con la geoquímica del azufre, es por ello que en la corteza terrestre, el principal mineral de Mo es la molibdenita $\left(\mathrm{MoS}_{2}\right)$. También se puede encontrar en otros minerales, como la wulfenita $\left(\mathrm{PbMoO}_{4}\right)$ y la powellita $\left(\mathrm{CaMoO}_{4}\right)$.

Bioquímicamente, el Mo llama la atención porque es un cofactor enzimático (coMo) esencial en casi todos los organismos (Paulsen et al. 1991, Cole et al. 1993, Marino et al. 2003, Tuit et al. 2004). Como cofactor enzimático tiene la función de asistir la transferencia de electrones en las oxotransferasas y promover la fijación de nitrógeno molecular en las nitrogenasas.

Tabla 1

Propiedades atómicas y físicas del Mo

Atomic and physical properties of the Mo

\begin{tabular}{|c|c|}
\hline Propiedades del Mo & Valores \\
\hline Número atómico, serie química & 42 , segunda serie de transición \\
\hline Configuración electrónica & {$[\mathrm{Kr}] 4 \mathrm{~d}^{5} 5 \mathrm{~s}^{1}$} \\
\hline Estados de oxidación & $+2,+3,+4,+6$ \\
\hline Densidad, dureza Mohs & $10280 \mathrm{~kg} \mathrm{~m}^{-3}, 5,5$ \\
\hline Masa atómica & $95,94 \mathrm{~g} \mathrm{~mol}^{-1}$ \\
\hline Radio covalente & $145 \mathrm{pm}$ \\
\hline Estructura cristalina & Cúbico centrado en el cuerpo \\
\hline Punto de fusión & $2623^{\circ} \mathrm{C}$ \\
\hline Punto de ebullición & $4639^{\circ} \mathrm{C}$ \\
\hline
\end{tabular}




\section{Geoquímica sedimentaria del molibdeno}

El Mo se encuentra en concentraciones traza en la corteza superior, en un rango de 1-2 ppm (Taylor \& McLennan 1985). La distribución de Mo en el ambiente depende de la especiación química en la que se encuentre, incluyendo su estado de oxidación (Morford \& Emerson 1999). En términos de la química de coordinación, el Mo es uno de los pocos metales de transición que forman oxoaniones y se coordinan solo débilmente con otros ligandos comunes en el ambiente sedimentario, tales como $\mathrm{Cl}^{-}$y $\mathrm{OH}^{-}$. Se encuentra asociado con $\mathrm{Cr}$ y W, así como también con Tc, Re, Os y U. De ahí, que la química del Mo tiene algunas analogías con estos metales, así como con los no-metales: S, Se, Py As, que también forman oxoaniones (Anbar 2004).

En términos de su química redox, el Mo se diferencia significativamente del $\mathrm{Cr}, \mathrm{U}$ y de muchos otros elementos, debido a que alcanza rápidamente su estado de oxidación más alto, $\mathrm{Mo}(\mathrm{VI})$, en soluciones con un potencial de óxido-reducción mayor a cero $(\mathrm{Eh}>0 \mathrm{~V})$ y un $\mathrm{pH}$ mayor a $5(\mathrm{pH}>5)$ (Fig. 1). Por consiguiente, la especiación de Mo en la mayoría de las aguas oxigenadas está dominada

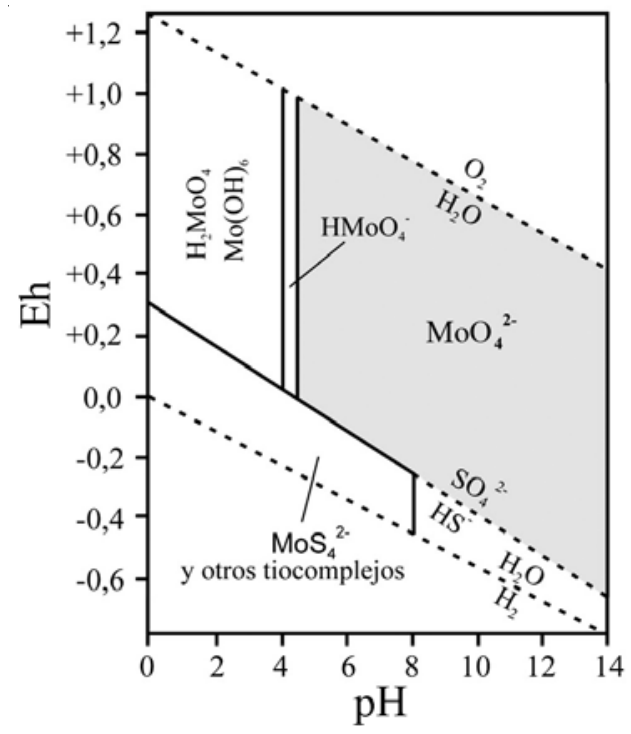

Figura 1

Diagrama Eh-pH para la especiación de Mo en el sistema Mo- $\mathrm{H}_{2} \mathrm{O}-\mathrm{S}$. $\Sigma \mathrm{Mo}=10^{-6} \mathrm{M} ; \Sigma \mathrm{S}=10^{-4} \mathrm{M}$. Se resalta la zona de la especie más abundante en el agua de mar, $\mathrm{MoO}_{4}{ }^{2-}$ (modificado de Manheim \& Landergren 1978)

Eh-pH diagram of dissolved Mo speciation in the system Mo$\mathrm{H}_{2} \mathrm{O}-\mathrm{S}$. $\Sigma \mathrm{Mo}=10^{-6} \mathrm{M} ; \Sigma \mathrm{S}=10^{-4} \mathrm{M}$. The zone with the most abundant species in the seawater is emphasized, $\mathrm{MoO}_{4}{ }^{2-}$ (modified after Manheim \& Landergren 1978) por el oxoanión de coordinación tetraédrica $\mathrm{MoO}_{4}{ }^{2-}$. En ambientes sedimentarios marinos, las especies protonadas tales como $\mathrm{HMoO}_{4}^{-}$o $\mathrm{H}_{2} \mathrm{MoO}_{4}$ (ácido molíbdico) no son cuantitativamente importantes, ya que rara vez se encuentran $\mathrm{pH}<6$ (Fig. 1). Las especies polinucleares de Mo, tal como $\mathrm{Mo}_{7} \mathrm{O}_{24}{ }^{6-} \mathrm{o}_{\mathrm{Mo}_{8}} \mathrm{O}_{26}{ }^{4-}$, son observadas a $\mathrm{pH}<6$, pero sólo en concentraciones altas $\left(>10^{-3} \mathrm{M}\right)$ son relevantes en el ambiente sedimentario (Erickson \& Helz 2000, Anbar 2004).

El comportamiento geoquímico de las especies tetraoxomolibdato (II) $\mathrm{MoO}_{4}^{2-}$ y sulfato $\left(\mathrm{SO}_{4}^{2-}\right)$ en sistemas oxigenados es semejante; ambas especies son transferidas en solución durante la meteorización, siendo muy móviles en fluidos oxidantes, y relativamente poco reactivas en los océanos. Las similitudes entre ambas especies se debe a que presentan características químicas similares, tales como: carga, coordinación y radios iónicos.

En la Fig. 2 se observa la distribución vertical de la concentración de $\mathrm{H}_{2} \mathrm{~S}$ y Mo en el Mar Negro (Emerson \& Huested 1991). Aquí, las aguas superficiales oxigenadas se hacen anóxicas con $\left[\mathrm{H}_{2} \mathrm{~S}\right]>100 \mu \mathrm{mol} \mathrm{kg}{ }^{-1}$ por debajo

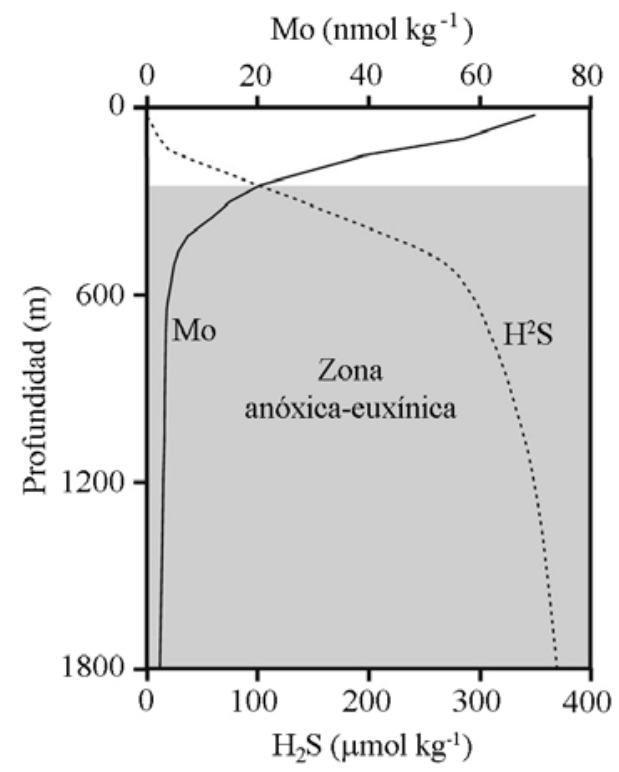

Figura 2

Distribución vertical de la concentración de $\mathrm{Mo} \mathrm{y} \mathrm{H}_{2} \mathrm{~S}$ en la Cuenca del Mar Negro (Mo: Emerson \& Huested 1991, $\mathrm{H}_{2} \mathrm{~S}$ : Neretin et al. 2001). La concentración de Mo baja bruscamente en la zona anóxica-euxínica

Depth profiles of Mo and $\mathrm{H}_{2} \mathrm{~S}$ in the Black Sea (Mo: Emerson \& Huested 1991, $\mathrm{H}_{2} \mathrm{~S}$ : Neretin et al. 2001). MO concentration falls sharply in the anoxic-euxinic zone 
de $\sim 100 \mathrm{~m}$. La concentración total de Mo a través de esta transición redox disminuye de $\sim 40 \mathrm{nmol} \mathrm{kg}^{-1}$ en la

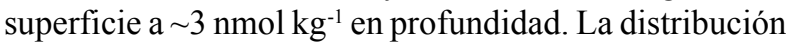
de Mo en los sedimentos marinos y aguas intersticiales indican que este elemento también se moviliza en las zonas intersticiales bajo condiciones reductoras intensas (Neubert et al. 2008). En general, la acumulación de Mo autigénico ocurre en sedimentos soterrados por aguas en las cuales la $\left[\mathrm{O}_{2}\right]<5 \mu \mathrm{mol} \mathrm{kg}{ }^{-1}$ (Emerson \& Huested 1991, Crusius et al. 1996, Zheng et al. 2000, Neubert et al. 2008).

El Mo no es concentrado por el plancton ordinario, y tampoco es adsorbido fácilmente por la mayoría de las partículas naturales (e.g. minerales de arcillas, carbonato de calcio, y oxihidróxidos de $\mathrm{Fe}$ ) en condiciones de $\mathrm{pH}$ marino 8-8,4 (Brumsack 1989, Goldberg et al. 1998). Por el contrario, este elemento es capturado fácilmente por oxidróxidos de $\mathrm{Mn}$, generalmente en la superficie de los sedimentos (Calvert \& Pedersen 1993, Crusius et al. 1996, Erickson \& Helz 2000, Zheng et al. 2000). La reducción subsecuente de estas fases libera el Mo adsorbido en las aguas intersticiales de los sedimentos (Crusius et al. 1996). Este fenómeno puede explicar el enriquecimiento de Mo durante el soterramiento; los óxidos de Mn presente en los sedimentos tienen el potencial de adsorber y de concentrar la especie $\mathrm{MoO}_{4}{ }^{2-}$ en o cerca de la interfase agua-sedimento (Adelson et al. 2001). Aunque este proceso puede concentrar Mo, no explica el mecanismo de fijación de Mo en los sedimentos. Por ello, es necesaria la reducción posterior para fijar este metal (Calvert \& Pedersen 1993, Crusius et al. 1996, Zheng et al. 2000).

Los modelos propuestos en la literatura buscan explicar el papel que desempeñan los sulfuros en la fijación del Mo en los sedimentos; por ejemplo, Helz et al. (1996) y Vorlicek et al. (2004) sugieren que los sulfuros disueltos en la columna de agua no sólo fijan el Mo a través de la formación de las especies $\mathrm{MoS}_{2} \mathrm{y} \mathrm{MoS}_{3}$, sino que propician la mineralización a través de la generación de intermediarios tiomolibdatos, así como complejos inorgánicos tipo 'cluster' que involucran Fe, Mo y S, y que posiblemente se encuentran en soluciones sólidas de sulfuros de Fe.

Helz et al. (1996) introdujeron el concepto de interruptor geoquímico, a través del cual la alta concentración de $\mathrm{H}_{2} \mathrm{~S} / \mathrm{HS}^{-}$en ambientes marinos euxínicos transforma el Mo de una especie química conservativa (molibdato: $\mathrm{MoO}_{4}{ }^{2-}$ ) a una reactiva (tiomolibdato: $\left.\mathrm{MoO}_{\mathrm{x}} \mathrm{S}_{4-\mathrm{x}}, \mathrm{x}=0-3\right)$. Los átomos de oxígeno en $\mathrm{MoO}_{4}{ }^{2-}$ son susceptibles de reemplazo por ligandos blandos, como donadores de S. Según Erickson \& Helz (2000), una etapa clave en este mecanismo inorgánico es la conversión de molibdato a tiomolibdato $\left(\mathrm{MoO}_{4}{ }^{2-} \mathrm{a} \mathrm{MoO}_{\mathrm{x}} \mathrm{S}_{4-\mathrm{x}}, \mathrm{x}=0-3\right)$, debido a la alta reactividad de estas especies químicas. $\mathrm{El} \mathrm{H}_{2} \mathrm{~S}$ es el reactivo limitante en esta reacción (Erickson \& Helz 2000, Zheng et al. 2000), ya que cada reacción sucesiva de sulfuración es aproximadamente un orden de magnitud más lenta que la anterior - ditiomolibdato $\leftarrow \rightarrow$ tritiomolibdato y tritiomolibdato $\leftarrow \rightarrow$ tetratiomolibdato; en consecuencia, estos equilibrios no se podrían alcanzar bajo condiciones intermitentes de aguas euxínicas (Erickson \& Helz 2000). Por otra parte, en los sedimentos, las reacciones de transformación son catalizadas por donadores de protones o por la presencia de algunos minerales con superficies activas, como por ejemplo caolinita (Erickson \& Helz 2000, Vorlicek \& Helz 2002).

Una vez formado el tiomolibdato, esta especie es removida de la columna de agua mediante quimisorción en la superficie de granos detríticos que contienen elementos metálicos (en particular hierro), o moléculas orgánicas sulfuradas (Helz et al. 1996, Vorlicek et al. 2004, Tribovillard et al. 2004). Los trabajos de Helz et al. (1996) y Bostick et al. (2003) sugieren que los 'cluster' monocristalinos de Fe-Mo-S que se han formado se preservan a través del tiempo geológico sobre la superficie de la pirita $\left(\mathrm{FeS}_{2}\right)$.

Hay que destacar que las partículas orgánicas también movilizan Mo en el ambiente sedimentario. La materia orgánica es un portador de Fe y otros metales traza. Los grupos orgánicos $\mathrm{O}-\mathrm{S}$ unidos a partículas macromoleculares pueden enlazar directamente $\mathrm{MoO}_{4}{ }^{2-}$, resultando un enlace covalente entre el elemento y la macromolécula. Helz et al. (1996) también resaltan el papel de los tioles provenientes de sustancias húmicas como activadores de Mo.

Como el Mo está presente en las nitrogenasas, una enzima usada por las cianobacterias para fijar nitrógeno, algunos investigadores (Paulsen et al. 1991, Cole et al. 1993, Marino et al. 2003, Tuit et al. 2004) han destacado que los flujos de estos elementos como micronutrientes son muy pequeños, por lo que es probable que su presencia en la materia orgánica no influya en los patrones de concentración de Mo en el ambiente sedimentario.

\section{Distribución del molibdeno en ambientes marinos}

Independientemente de la incertidumbre que se tenga sobre los mecanismos de remoción de Mo en el ambiente sedimentario marino, la baja concentración de oxígeno en la columna de agua es un factor crítico en la disponibilidad de este elemento en el ambiente sedimentario; esto explica por qué entre un 10 y 50\% del 
Mo que entra en los océanos, por el transporte de los ríos cada año, es removido de la columna de agua (Morford \& Emerson 1999). Gran parte de esta remoción ocurre en ambientes euxínicos, por ejemplo, Mar Negro y Cuenca de Cariaco (Arnold et al. 2004). De ahí que el Mo se encuentre enriquecido en lutitas negras, saprolitos y otros sedimentos carbonosos, con concentraciones entre 10$100 \mathrm{ppm}$. Aproximadamente entre el 10 y $20 \%$ de remoción de Mo también puede ocurrir en ambientes subóxicos que poseen un potencial redox intermedio, donde la $\left[\mathrm{O}_{2}\right]<5 \mu \mathrm{mol} \mathrm{kg}{ }^{-1}$ y la $\left[\mathrm{H}_{2} \mathrm{~S}\right]<<10 \mu \mathrm{mol} \mathrm{kg}{ }^{-1}$ (Morford \& Emerson 1999).

En vista a la estabilidad del $\mathrm{MoO}_{4}{ }^{2-}$ en solución, el Mo se encuentra enriquecido (concentraciones de cientos a miles de ppm) en óxidos de Fe-Mn (costras y nódulos) y en algunos sedimentos pelágicos óxicos. Tal enriquecimiento es el reflejo de la acumulación autigénica de Mo por adsorción y/o co-precipitación en minerales de óxidos de Mn. Este fenómeno se ha confirmado en experimentos de laboratorio realizadas por Barling \& Anbar (2004). Por lo tanto, la mayor parte del Mo que no es movilizado de los sedimentos en ambientes euxínicos, es removido en asociación con óxidos de Mn. Hay que destacar, que la concentración de Mo asociado a óxidos de $\mathrm{Mn}$ diseminados en sedimentos pelágicos puede ser cuantitativamente más importante, que el Mo que se encuentra en costras y nódulos de Fe-Mn, debido a que estos últimos se acumulan muy lentamente (Anbar 2004).

El Mo disuelto en aguas fluviales, al entrar en contacto con el ambiente sedimentario marino, es adsorbido por óxidos autigénicos de $\mathrm{Fe}-\mathrm{Mn}$ y sedimentos anóxicoseuxínicos (Fig. 3). La acumulación de este elemento en los océanos en relación con otros metales de transición refleja el fuerte carácter bimodal de la geoquímica del Mo con respecto a las condiciones redox. El Mo es fácilmente transferido de la corteza a los océanos por procesos de meteorización. Por el contrario, su tiempo de residencia en los océanos se encuentra alrededor de $800 \mathrm{ka}$, lo que muestra que el Mo es removido lentamente de los océanos. El Re y U tienen comportamientos geoquímicos similares a los del Mo (Anbar et al. 1992, Crusius et al. 1996). Una consecuencia de este comportamiento geoquímico es que la concentración del Mo en sedimentos (así como la concentración de $\mathrm{U}$ y Re) puede utilizarse como indicador local de condiciones redox en los fondos marinos (e.g. Crusius et al. 1996, Zheng et al. 2000, Sageman et al. 2003, Böning et al. 2009). En términos generales, un cambio en la concentración de Mo en los sedimentos de 10 a 100 ppm puede estar indicando bajos valores del potencial de

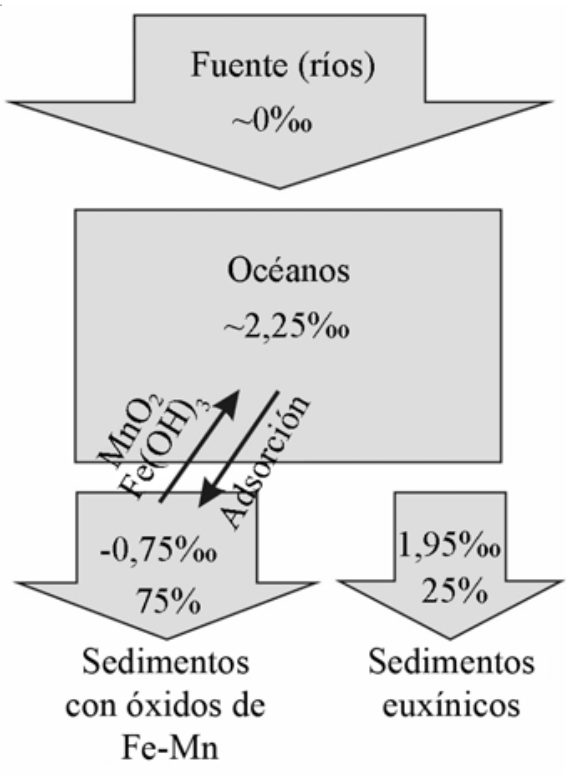

Figura 3

Distribución esquemática de la composición isotópica de Mo en los océanos modernos (Anbar 2004). El modelo no toma en cuenta los sedimentos depositados en condiciones subóxicos, y desiertos costeros donde el aporte eólico pudiera ser significativo. También se indican los valores isotópicos ( $\left.\delta^{98 / 95} \mathrm{Mo}\right)$ de entrada y salida, junto con el porcentaje de fraccionamiento de cada tipo de sedimento luego de la remoción del Mo de la columna de agua

Schematic depiction of the apparent Mo isotope budget in the modern oceans (Anbar 2004). The model does not take into account the sediments deposited in suboxic conditions, and coastal deserts where the aeolian contribution could be significant. Mean isotopic values $\left(\delta^{98 / 95} \mathrm{Mo}\right)$ for inputs and outputs are also indicated, along with the fractional

percentage of each sediment type for Mo removal in the water column

óxido-reducción (Eh) en la columna de agua durante la depositación de los sedimentos.

\section{Isótopos de molibdeno}

El molibdeno tiene siete isótopos estables $\left({ }^{92} \mathrm{Mo},{ }^{94} \mathrm{Mo}\right.$, ${ }^{95} \mathrm{Mo},{ }^{96} \mathrm{Mo},{ }^{97} \mathrm{Mo},{ }^{98} \mathrm{Mo},{ }^{100} \mathrm{Mo}$ ) con abundancia natural entre 10 y $25 \%$, un rango de masa alrededor del $8 \%$, y una vida media de $10^{19}$ años. Su comportamiento bajo condiciones reductoras y su facilidad para formar enlaces de tipo covalente, son las principales características que permiten explicar el fraccionamiento isotópico del Mo en el ambiente sedimentario marino, donde este metal se encuentra en forma de ión molibdato, $\mathrm{MoO}_{4}{ }^{2-}$. Bajo condiciones marinas oxidantes, el Mo es adsorbido 
lentamente de la columna de agua por óxidos autigénicos de Fe-Mn. Esta adsorción produce un fraccionamiento preferencial de los isótopos más livianos del Mo en los sedimentos ( $\delta^{98 / 95} \mathrm{Mo} \sim-0,7 \%$ o), quedando la columna de agua enriquecida en sus isótopos pesados (e.g. $\delta^{98 / 95} \mathrm{Mo}_{\text {agua }}$ de $\operatorname{mar} \sim 2,3 \%$ ). Por el contrario, bajo condiciones anóxicaseuxínicas (ricas en $\mathrm{H}_{2} \mathrm{~S}$ ), el Mo disuelto reacciona para formar tiomolibdatos $\left(\mathrm{MoO}_{\mathrm{x}} \mathrm{S}_{4-\mathrm{x}}, \mathrm{x}=0-3\right)$, sin ningún fraccionamiento isotópico (Tossell 2005). En consecuencia, la composición isotópica del Mo depositado bajo estas condiciones representa la composición isotópica del Mo en el agua de mar; en ambientes euxínicos modernos el $\delta^{98 / 95} \mathrm{Mo}_{\text {euxínico }} \approx \delta^{98 /}$ ${ }^{95} \mathrm{Mo}_{\text {agua de mar }} \approx 2,3 \%$ (Siebert et al. 2003, Arnold et al. 2004).

El fraccionamiento isotópico del Mo puede ser determinado mediante técnicas analíticas e instrumentales tales como la espectrometría de masa multicolector con fuente de ionización de plasma inductivamente acoplado ('MC-ICP-MS'). Una descripción detallada de la metodología analítica e instrumental para la determinación de la composición isotópica del Mo en muestras geológicas se discuten en Ambar (2004), Malinovsky et al. (2005), entre otros.

Las relaciones isotópicas de Mo utilizadas frecuentemente en la literatura (e.g. Anbar 2004, Siebert et al. 2003, Pearce et al. 2008, Neubert et al. 2008) son: $\delta^{97 / 95} \mathrm{Mo} \mathrm{y} \delta^{98 / 95} \mathrm{Mo}$; estas relaciones se expresan en partes por mil (\%) en relación al patrón estándar utilizado. En este trabajo nosotros usamos la relación $\delta^{98 / 95} \mathrm{Mo}$, pero sin embargo la relación $\delta^{97 / 95}$ Mo puede ser obtenida multiplicando por $0,67(2 / 3)$.

\section{Reservorios de isótopos de molibdeno}

En la Tabla 2 y Fig. 4 se muestra una compilación de datos de isótopos de Mo $\left(\delta^{98 / 95} \mathrm{Mo}\right)$ publicados en la literatura (McManus et al. 2002, Siebert et al. 2003, Barling et al. 2001, Anbar 2004, Arnold et al. 2004). En esta compilación de datos (Tabla 2), los valores de $\delta^{98 /}$ ${ }^{95} \mathrm{Mo}$ en rocas ígneas, fluido hidrotermal, molibdenitas y sedimentos clásticos varían entre $-0,4-0,36 \%$. Los valores de $\delta^{98 / 95} \mathrm{Mo}$ de sedimentos euxínicos holocenos se encuentran entre 1,67 - 2,55\%. Por el contrario, los $\delta^{98 / 95}$ Mo en sedimentos depositados bajo condiciones óxicas: óxidos de Fe-Mn (costras y nódulos superficiales) y sedimentos pelágicos, son menos pesados y varían entre $-0,8$ y $-0,3 \%$. Globalmente, los valores de $\delta^{98 / 95}$ Mo de sedimentos euxínicos holocenos presentan un promedio $\sim 2,2 \%$, dos órdenes de magnitud mayor que los valores de $\delta^{98 / 95}$ Mo en sedimentos óxicos, lo que pone en evidencia su aplicabilidad como marcador de condiciones redox.

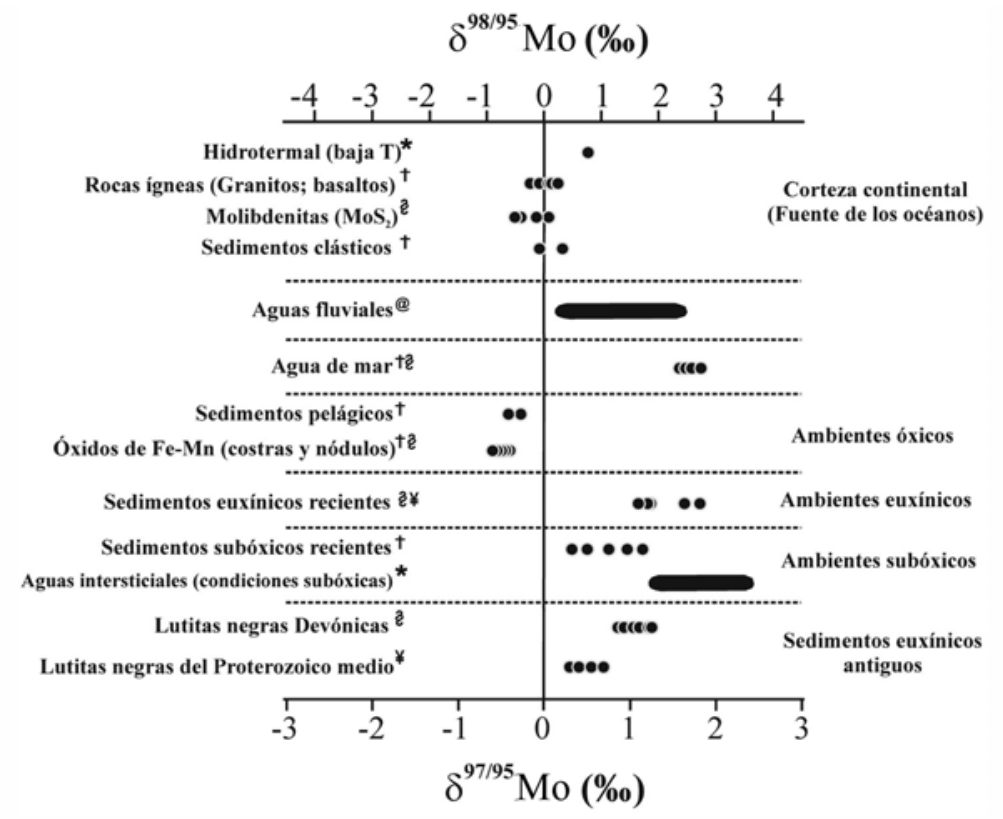

(*) McManus et al. 2002, ( $($ ) Siebert et al. 2003, (§) Barling et al. 2001, (¥) Amold et al. 2004, (@)Archer \& Vance 2008

Figura 4

Fraccionamiento isotópico del Mo en muestras naturales. $\delta^{97 / 95} \mathrm{Mo} \sim 2 / 3 \times$ ä$^{98 / 95}$ Mo

The isotopic fractionation of Mo data from natural samples. $\delta^{97 / 95} \mathrm{Mo} \sim 2 / 3 \times \ddot{a}^{98 / 95} \mathrm{Mo}$ 
Tabla 2

Compilación de datos de concentraciones de Mo y su fraccionamiento isotópico $\left(\delta^{98 / 95} \mathrm{Mo}\right)$ en muestras naturales

Data compilation of Mo concentrations and its isotopic fractionation $\left(\delta^{98 / 95} \mathbf{M o}\right)$ from natural samples

\begin{tabular}{|c|c|c|}
\hline & Mo (ppm) & $\delta^{98 / 95} \mathrm{Mo}^{*}$ \\
\hline \multicolumn{3}{|l|}{ Fluido hidrotermal (baja temperatura) } \\
\hline Baby Bare Hydrothermal Spring ${ }^{a}$ & $297 \mathrm{nmol} \mathrm{kg}^{-1}$ & $\sim 0,8$ \\
\hline \multicolumn{3}{|l|}{ Rocas ígneas } \\
\hline Granitos (103B, Himalaya) ${ }^{\mathrm{b}}$ & $12,1-19,1$ & $0,10-0,14$ \\
\hline Basaltos (Kamchatka) ${ }^{\mathrm{b}}$ & $0,7-1,4$ & $-0,09-0,25$ \\
\hline \multicolumn{3}{|l|}{ Molibdenitas } \\
\hline $34844,34843,34840$ (Nambucca, Australia) $^{\mathrm{c}}$ & - & $-0,06--0,39$ \\
\hline HLP-5 (Huanglongpu, China) ${ }^{\mathrm{c}}$ & - & $-0,26--0,40$ \\
\hline \multicolumn{3}{|l|}{ Sedimentos clásticos } \\
\hline GUA-4, FON-1 (España) ${ }^{\text {b }}$ & $1,2-2,7$ & $0,00-0,36$ \\
\hline \multicolumn{3}{|l|}{ Aguas fluviales } \\
\hline $\begin{array}{l}\text { Amazonas, Nilo, Brahmaputra, Chang Jiang, Kalix, } \\
\text { Volga, Ottawa }^{\mathrm{e}}\end{array}$ & $2-18,1 \mathrm{nmol} \mathrm{kg}^{-1}$ & $0,15-2,40$ \\
\hline \multicolumn{3}{|l|}{ Agua de mar } \\
\hline Atlántico ${ }^{\mathrm{b}}$ & 0,011 & 2,3 \\
\hline Índico $^{b}$ & 0,011 & 2,3 \\
\hline Pacífico ${ }^{\mathrm{b}, \mathrm{c}}$ & 0,011 & 2,4 \\
\hline Mar de Labrador ${ }^{\mathrm{b}}$ & 0,011 & 2,5 \\
\hline \multicolumn{3}{|l|}{ Sedimentos pelágicos (Cuaternario) } \\
\hline $53-45-2\left(11^{\circ} 44^{\prime} \mathrm{N}, 138^{\circ} 22,2^{\prime} \mathrm{W}\right)^{\mathrm{b}}$ & 3,4 & $-0,30$ \\
\hline $48-19-17\left(08^{\circ} 16,5^{\prime} \mathrm{N}, 151^{\circ} 07,3^{\prime} \mathrm{W}\right)^{\mathrm{b}}$ & 185 & $-0,50$ \\
\hline \multicolumn{3}{|l|}{ Óxidos de Fe-Mn (costras y nódulos superficiales) } \\
\hline Pacífico $^{\mathrm{b}}$ & $277-459$ & $-0,5--0,7$ \\
\hline Índico ${ }^{\mathrm{b}}$ & $296-496$ & $-0,6--0,7$ \\
\hline Atlántico ${ }^{\mathrm{b}}$ & $239-307$ & $-0,7--0,8$ \\
\hline \multicolumn{3}{|l|}{ Sedimentos euxínicos recientes } \\
\hline Cuenca de Cariaco (ODP leg 165) ${ }^{\mathrm{d}}$ & $150-180$ & $1,64-1,94$ \\
\hline Mar Negro (Unidad 1 STA9) ${ }^{\mathrm{d}}$ & 16 & $2,51-2,55$ \\
\hline \multicolumn{3}{|l|}{ Sedimentos subóxicos recientes } \\
\hline Cuenca San Pedro ${ }^{\mathrm{b}}$ & $3,4-4,0$ & $0,52-0,72$ \\
\hline Cuenca Santa Mónica $^{\mathrm{b}}$ & 4,9 & 1,11 \\
\hline Margen continental de Chile ${ }^{\mathrm{b}}$ & $40,8-131,9$ & $1,62-1,39$ \\
\hline \multicolumn{3}{|l|}{ Aguas intersticiales (condiciones subóxicas) } \\
\hline Cuenca Santa Mónica ${ }^{\mathrm{a}}$ & $75-190 \mathrm{nmol} \mathrm{kg}^{-1}$ & $1,90-3,50$ \\
\hline \multicolumn{3}{|l|}{ Lutitas negras (Devónico) } \\
\hline Devonian Ohio Shale (USGS SDO-1) ${ }^{\mathrm{c}}$ & $113-155$ & $1,23-1,74$ \\
\hline \multicolumn{3}{|l|}{ Lutitas negras (Proterozoico medio) } \\
\hline Formación Velkerri (northern Australia) ${ }^{\mathrm{d}}$ & $11-60$ & $0,47-1,01$ \\
\hline Formación Wollogorang (northern Australia) ${ }^{\mathrm{d}}$ & $42-52$ & $0,62-0,99$ \\
\hline
\end{tabular}

: $\delta^{9899} \mathrm{Mo}=\left[\left({ }^{98} \mathrm{Mo} /{ }^{95} \mathrm{Mo}\right)\right.$ muestra $/\left({ }^{98} \mathrm{Mo} /{ }^{95} \mathrm{Mo}\right)$ estándar $\left.)-1\right] \times 1000$; a: McManus et al. 2002; b: Siebert et al. 2003; c: Barling et al. 2001; d: Arnold et al. 2004; e: Archer \& Vance 2008 
A continuación se discuten las características isotópicas más resaltantes de estos reservorios naturales de molibdeno.

\section{Rocas ígneas (granitos y basaltos)}

Los datos de isótopos de Mo en rocas ígneas son escasos. Sin embargo, Siebert et al. (2003) analizaron muestras de basaltos y granitos de edad Arqueana y Terciaria, obteniendo como resultado un intervalo estrecho de isótopos de Mo. Un granito Arqueano y un granito Terciario del Himalaya tienen semejantes valores de $\delta^{98 /}$ ${ }^{95} \mathrm{Mo}$ alrededor de $0 \%$. Los basaltos de subducción de Kamchatka (Plioceno-Pleistoceno) tienen $\delta^{98 / 95} \mathrm{Mo}$ similares. Estos resultados sugieren que el fraccionamiento isotópico del Mo durante los procesos ígneos es pequeño comparado con los límites analíticos actuales (Siebert et al. 2003).

\section{2. Óxidos de Fe-Mn (costras y nódulos)}

La distribución de isótopos de Mo en óxidos de Fe-Mn ha sido estudiada por algunos autores (e.g. Siebert et al. 2003, Barling \& Anbar 2004). Estas investigaciones están basadas en estudios de muestras de nódulos de los océanos Pacífico y Atlántico, y costras de los océanos Pacífico, Atlántico e Indico. La concentración de Mo varía entre 277 a 493 ppm y las composiciones isotópicas del Mo son considerablemente livianas y uniformes en todas las muestras. Los $\delta^{98 / 95}$ Mo en estas muestras se encuentran alrededor de - 1 y $-0,5 \%$. No hay diferencias significativas entre la composición isotópica de los nódulos y las costras a pesar de las diferencias de acumulación de estos óxidos (Anbar 2004).

\section{Sedimentos marinos}

A.- Sedimentos euxínicos. La característica más significativa de los isótopos de Mo en estos ambientes es su enriquecimiento en $\delta^{98 / 95} \mathrm{Mo}$, específicamente en el isótopo ${ }^{98} \mathrm{Mo}$ (Barling et al. 2001, Arnold et al. 2004). Los sedimentos holocenos ricos en materia orgánica, del Mar Negro y Cuenca de Cariaco, presentan un $\delta^{98 / 95} \mathrm{Mo}$ mayores al $1,5 \%$, por lo que hay una diferencia significativa entre las magnitudes de $\delta^{98 / 95} \mathrm{Mo}$ de estos sedimentos con respecto a las magnitudes en rocas ígneas y óxidos de Fe-Mn. A diferencia de los casos anteriores, los valores de $\delta^{98 / 95} \mathrm{Mo}$ entre los sedimentos euxínicos se encuentran ligeramente dispersos.

B.- Sedimentos subóxicos. Siebert et al. (2003) estudiaron la distribución de los isótopos de Mo en un número limitado de muestras de sedimentos marinos acumulados bajo condiciones subóxicas o disóxicas $\left(\left[\mathrm{O}_{2}\right]<5 \mu \mathrm{mol} \mathrm{kg}^{-1}\right)$. Las concentraciones de Mo en estos sedimentos están enriquecidas sobre el promedio de la corteza por un factor alrededor de 3-100. Isotópicamente, el $\delta^{98 / 95} \mathrm{Mo}$ en estos sedimentos cubre un intervalo grande, cuyos deltas están entre las rocas ígneas y los sedimentos euxínicos.

C.- Sedimentos clásticos y pelágicos. Los isótopos de Mo en muestras de sedimentos clásticos y pelágicos han sido poco estudiados. Siebert et al. (2003) sugieren que los sedimentos pelágicos se encuentran enriquecidas en Mo (3,4-185 ppm), y empobrecidas en $\delta^{98 / 95} \mathrm{Mo}$ (semejante al delta de los óxidos de Fe-Mn). Por el contrario, los sedimentos clásticos tienen bajas concentraciones de Mo (1-3 ppm) y una composición isotópica de Mo indistinguible al de las rocas ígneas (Siebert et al. 2003).

\section{Aguas}

A.- Agua de mar. Existe una gran cantidad de datos sobre la distribución de isótopos de Mo en agua de mar (e.g. Barling et al. 2001, Siebert et al. 2003, Anbar 2004). Los análisis de isótopos de Mo en muestras de aguas recolectadas a más de $3000 \mathrm{~m}$ de profundidad en los océanos Pacífico, Atlántico e Índico, revelan dos características importantes: (a) no hay variaciones perceptibles en los $\delta^{98 / 95} \mathrm{Mo}$, y (b) los $\delta^{98 / 95}$ Mo son semejantes a los deltas de los sedimentos euxínicos, alrededor de 2,3-3\%. La uniformidad en la composición isotópica de Mo en el agua de mar es concordante con el largo tiempo de residencia de este metal (Siebert et al. 2003). Esta homogeneidad también puede ser constatada cuando se comparan las muestras de agua de mar con el patrón de referencia MOMO ('mean ocean molybdenum'), en este caso los $\delta^{98 / 95}$ Mo se encuentran alrededor de $0 \%$.

B.- Aguas fluviales. Archer \& Vance (2008) estudiaron el fraccionamiento de los isótopos de Mo en un conjunto de ríos a nivel mundial que representan el $22 \%$ de la descarga de agua de río total. Las concentraciones de Mo disuelto varían entre 2 y $18,1 \mathrm{nmol} \mathrm{kg}^{-1}$. Los resultados revelan una amplia variabilidad en la composición isotópica de $\mathrm{Mo}$ en estos ríos $\left(\delta^{98 / 95} \mathrm{Mo}=0,15-2,40 \%\right)$, encontrándose enriquecidos en isótopos pesados $\left({ }^{98} \mathrm{Mo}\right)$ en comparación con las rocas continentales $\left(\delta^{98 / 95} \mathrm{Mo} \sim\right.$ $0,09 \pm 0,24 \%$ ). Además los resultados también revelan que bajos valores en $\delta^{98 / 95} \mathrm{Mo}$ (e.g., $\sim 0,15 \%$ ) se asocian con altas concentraciones de Mo en solución, reflejando el promedio cortical de isótopos de Mo (granitos, basaltos, sedimentos clásticos). Por el contrario, altos valores de $\delta^{98 / 95} \mathrm{Mo}$ (e.g. $\sim 2,40 \%$ ) asociados con bajas concentraciones de Mo en solución, son generados por un fraccionamiento isotópico producido durante la 
meteorización y por retención en los suelos de los isótopos más livianos de Mo (Archer \& Vance 2008).

\section{C.- Fluidos hidrotermales de baja temperatura. Los} fluidos calientes que emergen de sistemas hidrotermales en dorsales oceánicas han surgido recientemente como una fuente neta de Mo y otros elementos en los océanos (Wheat \& Mottl 2000). Los isótopos de Mo en estos fluidos son más livianos que los del agua de mar, pero más pesados que los ígneos, presentando $\delta^{98 / 95} \mathrm{Mo}$ alrededores de 0,75\%o (McManus et al. 2002, Anbar 2004).

D.- Aguas intersticiales de sedimentos (condiciones subóxicas). Como se discutió anteriormente, los $\delta^{98 / 95}$ Mo en sedimentos subóxicos tienen magnitudes intermedias entre las rocas ígneas, sedimentos euxínicos y agua de mar. McManus et al. (2002) estudiaron los $\delta^{98 / 95}$ Mo en aguas intersticiales de núcleos de sedimentos de la Cuenca de Santa Mónica, oeste de los Estados Unidos (Fig. 5), donde la $\left[\mathrm{O}_{2}\right]<5 \mu \mathrm{mol} \mathrm{kg-1}$. Los $\delta^{98 / 95}$ Mo correspondientes a la matriz por debajo de la interfase agua-sedimento son alrededor de 0,25\%, más livianos que en el agua de mar, reflejando la liberación de Mo asociado a los óxidos de Mn. La concentración de Mo disminuye progresivamente a los $5 \mathrm{~cm}$ de profundidad, conjuntamente con esta

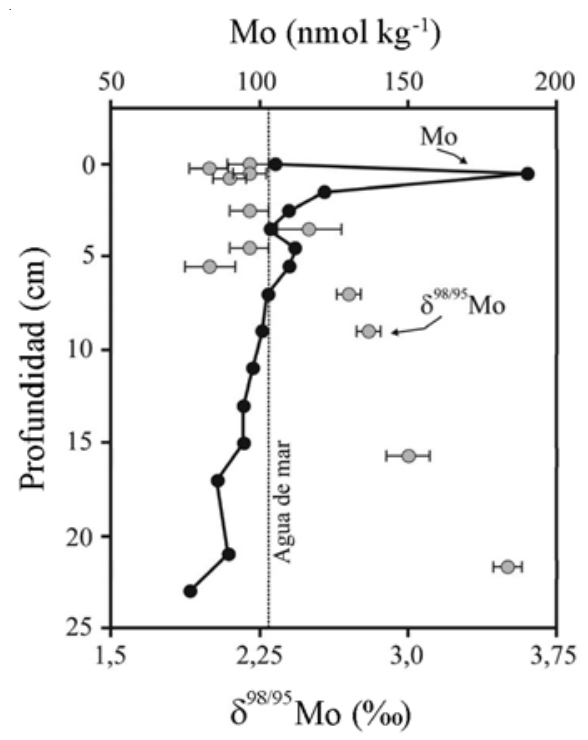

Figura 5

Concentración de Mo y composición isotópica ( a $\left.^{98 / 95} \mathrm{Mo}\right)$ en fluidos intersticiales reductores en la Cuenca Santa Mónica (McManus et al. 2002)

Mo concentrations and isotopic compositions ( $\left(\ddot{a}^{98 / 95} \mathrm{Mo}\right)$ from reducing pore fluids in Santa Monica Basin

(McManus et al. 2002) disminución, el $\delta^{98 / 95}$ Mo se enriquece progresivamente en ${ }^{98} \mathrm{Mo}$, llegando alcanzar un valor más pesado que el agua de mar por un factor de $1 \%$.

\section{Otras aplicaciones de los isótopos de molibdeno}

El amplio rango $(\sim 4 \%)$ en los valores de $\delta^{98 / 95} \mathrm{Mo}$ observados en los reservorios de Mo ofrece un número amplio de aplicaciones: análisis de cuencas de edades Fanerozóicas, intensidad de paleo-meteorización, variaciones locales en las condiciones reductoras de los océanos a través de la historia de la Tierra, y el aumento de los niveles de oxígeno atmosférico en la Tierra primitiva (e.g. Siebert et al. 2003, Anbar, 2004, Pearce et al. 2008, Archer \& Vance 2008).

Diversos modelos sugieren que la proporción del flujo de liberación de Mo en condiciones óxicas a anóxicas en los océanos modernos es aproximadamente de 7:3. La composición de isótopos de Mo se mantiene relativamente constantes en costras de óxidos de Fe-Mn a través del tiempo, lo cual indica que se han producido pequeñas variaciones en esta proporción durante los últimos 60 millones de años (Siebert et al. 2003).

Aun no se han publicado datos sobre la formación de costras de óxidos de Fe-Mn (Siebert et al. 2003) en tiempos pre-Cenozoicos, es por ello que la composición de isótopos de Mo determinada en un gran número de muestras de sedimentos de esta edad, pueden ser de gran utilidad para estudiar este fenómeno a nivel mundial.

Como se discutió anteriormente, el comportamiento geoquímico del Mo bajo condiciones anóxicas-euxínicas, es el aspecto principal que explica el fraccionamiento isotópico del Mo en el ambiente sedimentario marino. Por lo tanto, diversos autores (e.g. Anbar 2004, Archer \& Vance 2008) especulan que el fraccionamiento isotópico del Mo entre rocas corticales y sedimentos Arqueanos, debería ser insignificante debido a la persistencia de una atmósfera reductora durante este tiempo. Dentro este contexto, los isótopos de Mo podrían ser útiles para estudiar el aumento de oxígeno atmosférico en la atmósfera primitiva (Siebert et al. 2003).

\section{Alteración de la composición isotópica del molibdeno en secuencias sedimentarias antiguas}

Diversos autores (McManus et al. 2002, Siebert et al. 2003, Barling \& Anbar 2004, Williams et al. 2004), han estudiado la influencia de la diagénesis y meteorización sobre el registro geoquímico del Mo, incluyendo su composición isotópica, en secuencias sedimentarias. 
Pirita

Costras y nódulos de óxidos Fe-Mn

$\approx$ Fitoplancton

- Materia orgánica detrítica

$\square$ Materia orgánica sulfurada

- Materia orgánica metabolizable

X Especies químicas (Mo, U, V, Mn, Fe, Cu, Ni, Co, Al, Si)

disueltas en la columna de agua oxigenada (oxoaniones solubles)
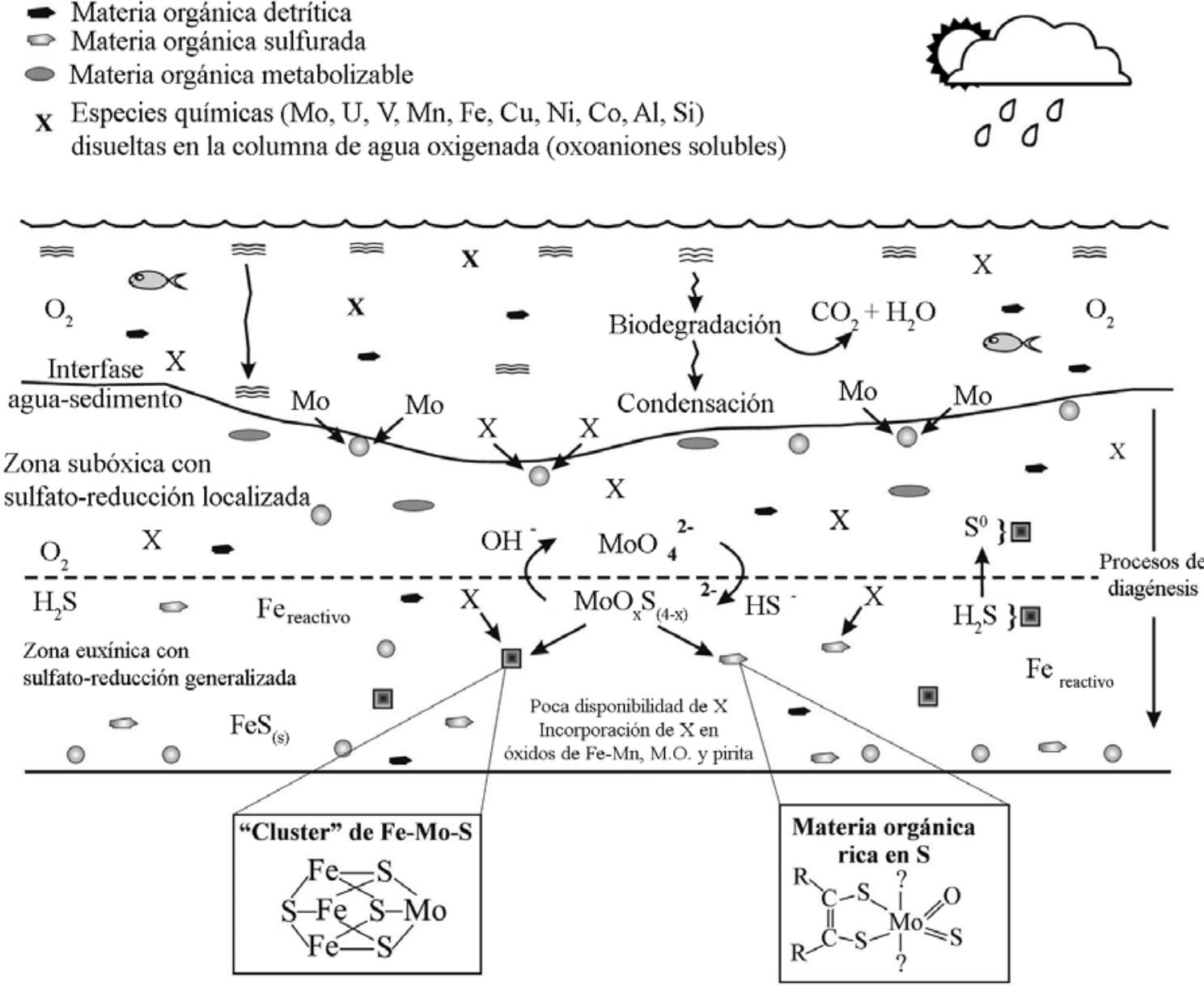

$\mathrm{S}^{0}$ \} mecanismo: $\mathrm{FeS}(\mathrm{s})+\mathrm{S}_{\mathrm{n}}^{2-}(\mathrm{aq}) \rightarrow \mathrm{FeS}_{2}(\mathrm{~s})+\mathrm{S}_{\mathrm{n}-1}^{2-}(\mathrm{aq})$

$\mathrm{H}_{2} \mathrm{~S}$ \} mecanismo: $\mathrm{FeS}(\mathrm{s})+\mathrm{H}_{2} \mathrm{~S}(\mathrm{aq}) \rightarrow \mathrm{FeS}_{2}(\mathrm{~s})+\mathrm{H}_{2}(\mathrm{~g})$

Figura 6

Modelo generalizado de la sedimentación del Mo en cuencas marinas anóxicas-euxínicas. En la zona euxínica, el $\mathrm{MoO}_{4}{ }^{2-}$ reacciona formando tiomolibdatos. Posteriormente, el Mo en los tiomolibdatos puede ser removido por partículas detríticas ricas en Fe, óxidos de Fe-Mn y por moléculas orgánicas ricas en azufre que capturan al Mo como oxitiomolibdatos orgánicos. Los productos sedimentarios de estos procesos son representados como 'cluster' de Fe-Mo-S y tiomolibdatos orgánicos

Generalized model of Mo sedimentation in anoxic-euxinic ocean basins. In the sulfidic zone, $\mathrm{MoO}_{4}{ }^{2-}$ begins to convert to thiomolybdates. Subsequently, thiomolydates can be scavenged by Fe-bearing detritus, Fe-Mn oxides (ferromanganese nodules) and sulfidized organic particles can capture Mo as organic oxythiomolybdates. The sedimentary products of these processes are represented here as Fe-Mo-S cluster and organic thiomolybdates 
Williams et al. (2004) estudiaron un perfil meteorizado de lutitas negras de edad Devónica cerca de Clay City, Kentucky (Estados Unidos), encontrando un aumento de $0,6 \%$ en el $\delta^{98 / 95} \mathrm{Mo}$ (en relación con el estándar Rochester JMC) cerca del horizonte meteorizado. Este cambio va acompañado por un aumento en la concentración de Mo y Mn, y una pérdida casi total de Re; el Re también es utilizado como un marcador de condiciones redox (e.g. Pearce et al. 2008). Esta coincidencia en los cambios de las concentraciones elementales y la composición isotópica sugieren que el Mo es adsorbido por óxidos de Mn diagenéticos. Estos autores indican que el $\delta^{98 / 95}$ Mo en las muestras de lutitas está alrededor de $0,45 \%$, sugiriendo condiciones de depositación disóxicas; este delta es considerablemente menos pesado que el de los sedimentos euxínicos modernos ( 2,3\%o) y otras lutitas Devónicas (1,5\%o). Sin embargo, ellos también destacan que el estudio local de marcadores redox, tales como el índice de piritización ('DOP') y la relación $\mathrm{Mo} / \mathrm{Al}$, permitieron determinar con menor incertidumbre que las condiciones de depositación en el ambiente sedimentario fueron predominantemente anóxicas y pobremente euxínicas.

Estos resultados demuestran que la oxidación diagenética puede alterar los paleo-registros de los isótopos de Mo; por ello se hace imperativo la combinación de datos de isótopos de Mo con otros marcadores redox (DOP, Mo/Al, U/Al, REEs, etc.) cuando se realizan estudios de las condiciones de paleoxigenación en ambientes sedimentarios marinos.

Finalmente, a partir de este resumen bibliográfico se elaboró un modelo conceptual del comportamiento geoquímico del molibdeno en el ambiente sedimentario marino (Fig. 6). Este modelo es una contribución esquemática por el esfuerzo de entender la geoquímica de este metal en ambientes marinos anóxicos-euxínicos.

\section{Conclusiones}

Este trabajo resume el comportamiento geoquímico del molibdeno y sus isótopos estables en el ambiente sedimentario. El alto tiempo de residencia de Mo en los océanos $(\sim 800 \mathrm{ka})$, las variaciones sistemáticas en la composición isotópica del Mo en los océanos y la preservación de sus huellas isotópicas en sedimentos marinos, lutitas negras, agua intersticial y en óxidos de Fe-Mn, evidencian la aplicabilidad del Mo y sus isótopos estables como marcadores de condiciones reductoras en ambientes sedimentarios marinos. No obstante, los procesos de meteorización y diagénesis pueden alterar los paleoregistros del Mo, por ello es necesario combinar este indicador elemental con otros marcadores redox tales como carbono orgánico total (COT), U, V, Re, tierras raras (REEs), entre otros.

Una mejor comprensión sobre el comportamiento geoquímico del molibdeno en registros sedimentarios modernos, permitiría realizar una calibración más robusta sobre el fraccionamiento isotópico del Mo en ambientes sedimentarios antiguos, y sus implicaciones como elemento químico sensible a las variaciones fisicoquímicas del ambiente sedimentario. Sin embargo, los estudios y datos disponibles en la literatura muestran claramente su utilidad en reconstrucciones paleoambientales.

\section{Agradecimientos}

Este trabajo fue realizado con el apoyo del Programa Alban, Programa de Becas de Alto Nivel de la Unión Europea para América Latina, beca No E06D100913VE. Agradecemos los comentarios y sugerencias de los evaluadores anónimos.

\section{Literatura citada}

Adelson JM, GR Helz \& CV Miller. 2001. Reconstructing the rise of recent coastal anoxia; molybdenum in Chesapeake Bay sediments. Geochimica et Cosmochimica Acta 65: 237252.

Algeo TJ \& TW Lyons. 2006. Mo-TOC covariation in modern anoxic marine environments: implication for analysis of paleoredox and hydrographic conditions. Paleoceanography 21: PA1016, <doi:10.1029/2004PA001112>

Anbar AD. 2004. Molybdenum stable isotopes: observations interpretations and directions. Reviews in Mineralogy and Geochemistry 55: 429-454.

Anbar AD, RA Creaser, DA Papanastassiou \& GJ Wasserburg. 1992. Rhenium in seawater: confirmation of generally conservative behavior. Geochimica et Cosmochimica Acta 56: 4099-4103.

Archer C \& D Vance. 2008. The isotopic signature of the global riverine molybdenum flux and anoxia in the ancient oceans. Nature Geosciences 1: 597-600.

Arnold GL, AD Anbar, J Barling \& TW Lyons. 2004. Molybdenum isotope evidence for widespread anoxia in Mid-Proterozoic oceans. Science 304: 87-90.

Barling $\mathbf{J}$ \& AD Anbar. 2004. Molybdenum isotope fractionation during adsorption by manganese oxides. Earth and Planetary Science Letters 217: 315-329.

Barling J, GL Arnold \& AD Anbar. 2001. Natural massdependent variations in the isotopic composition of molybdenum. Earth Planet Science Letter 193: 447-457.

Böning P, HJ Brumsack, B Schnetger \& M Grunwald. 2009. Trace element signatures of Chilean upwelling sediments at $\sim 36^{\circ} \mathrm{S}$. Marine Geology 259: 112-121. 
Bostick BC, S Fendorf \& GR Helz. 2003. Differential adsorption of molybdate and terathiomolybdate on pyrite $\left(\mathrm{FeS}_{2}\right)$. Environmental Science and Technology 37: 285291.

Brumsack HJ. 1989. Geochemistry of recent TOC-rich sediments from the Gulf of California and the Black Sea. Geological Rundsch 78: 851-882.

Calvert SE \& TF Pedersen. 1993. Geochemistry of recent oxic and anoxic sediments: implications for the geological record. Marine Geology 113: 67-88.

Cole JJ, J Lane, R Marino \& RW Howarth. 1993. Molybdenum assimilation by cyanobacteria and phytoplankton in freshwater and salt water. Limnology and Oceanography 38: 25-35.

Crusius J, S Calvert, T Pedersen \& D Sage. 1996. Rhenium and molybdenum enrichments in sediments as indicators of oxic, suboxic, and sulfidic conditions of deposition. Earth and Planetary Science Letters 145: 65-78.

Emerson SR \& SS Huested. 1991. Ocean anoxia and the concentration of molybdenum and vanadium in seawater. Marine Chemistry 34: 177-196.

Erickson BE \& GR Helz. 2000. Molybdenum(VI) speciation in sulfidic waters: stability and lability of thiomolybdates. Geochimica et Cosmochimica Acta 64: 1149-1158.

Goldberg S, C Su \& HS Forster. 1998. Sorption of Molybdenum on oxides, clay minerals, and soils: Mechanisms and models. En: Jenne EA(ed). Adsorption of metals by Geomedia, Chapter 19: 401-426. Academic Press, San Diego.

Helz GR, CV Miller, JM Charnock, JL Mosselmans, RA Pattrick, CD Garner \& DJ Vaughan. 1996. Mechanisms of molybdenum removal from the sea and its concentration in black shales: EXAFS evidences. Geochimica et Cosmochimica Acta 60: 3631-3642.

Malinovsky D, I Rodushkin, DC Baxter, J Ingri \& B Öhlander. 2005. Molybdenum isotope ratio measurements on geological samples by MC-ICPMS. International Journal of Mass Spectrometry 245: 94-107.

Manheim FT \& S Landergren. 1974. Molybdenum. En: Wedepohl KH (ed). Handbook of geochemistry, Vol. 2(3): 1-87. Springer-Verlag, New York.

Marino R, RW Howarth, F Chan, JJ Cole \& GE Likens. 2003. Sulfate inhibition of molybdenum-dependent nitrogen fixation by planktonic cyanobacteria under seawater conditions: a non-reversible effect. Hydrobiology 500: 277 293.

McManus J, TF Nagler, C Siebert, CG Wheat \& DE Hammond. 2002. Oceanic molybdenum isotope fractionation: Diagenesis and hydrothermal ridge-flank alteration. Geochemistry, Geophysics, Geosystems 3(12): 1078, < doi:10.1029/2002GC000356>
McManus J, WM Berelson, GP Klinkhammer, DE Hammond \& C Holm. 2005. Authigenic uranium: relationship to oxygen penetration depth and organic carbon rain. Geochimica et Cosmochimica Acta 69: 95-108.

Morford JL \& S Emerson. 1999. The geochemistry of redox sensitive trace metals in sediments. Geochimica et Cosmochimica Acta 63: 1735-1750.

Neretin LN, II Volkov, ME Böttcher \& VA Grinenko. 2001. A sulfur budget for the Black Sea anoxic zone. Deep Sea Research 48: 2569-2593.

Neubert N, TF Nägler \& ME Böttcher. 2008. Sulfidity controls molybdenum isotope fractionation into euxinic sediments: Evidence from the modern Black Sea. Geology 36: $775-778$.

Paulsen DM, HW Paerl \& PE Bishop. 1991. Evidence that molybdenum dependent nitrogen fixation is not limited by high concentrations of sulfate in marine environments. Limnology and Oceanography 36: 1325-1334.

Pearce CR, AS Cohen, AL Coe \& KW Burton. 2008. Molybdenum isotope evidence for global ocean anoxia coupled with perturbations to the carbon cycle during the early Jurassic. Geology 36: 231-234.

Poulson RL, C Siebert, J McManus \& WM Berelson. 2006. Authigenic molybdenum isotope signatures in marine sediments. Geology 34: 617-620.

Sageman BB, AE Murphy, JP Werne, CA ver Straeten, DJ Hollander \& TW Lyons. 2003. A tale of shales: the relative roles of production, decomposition, and dilution in the accumulation of organic-rich strata, Middle-Upper Devonian, Appalachian Basin. Chemical Geology 195: 229273.

Siebert C, TF Nagler, F von Blanckenburg \& JD Kramers. 2003. Molybdenum isotope records as a potential new proxy for paleoceanography. Earth and Planetary Science Letters 211: 159-171.

Tossell JA. 2005. Calculating the partitioning of the isotopes of Mo between oxidic and sulfidic species in aqueous solution. Geochimica et Cosmochimica Acta 69: 2981-2993.

Tribovillard N, A Riboulleau, T Lyons \& F Baudin. 2004. Enhanced trapping of molybdenum by sulfurized organic matter of marine origin as recorded by various Mesozoic formations. Chemical Geology 213: 385-401.

Tribovillard N, TJ Algeo, T Lyons \& A Riboulleau. 2006. Trace metals as paleoredox and paleoproductivity proxies: An update. Chemical Geology 232: 12-32.

Tuit C, J Waterbury \& G Ravizza. 2004. Diel variation of molybdenum and iron in marine diazotrophic cyanobacteria. Limnology and Oceanography 49: 978-990.

Vorlicek TP \& GR Helz. 2002. Catalysis by mineral surfaces: implications for Mo geochemistry in anoxic environments. Geochimica et Cosmochimica Acta 66: 3679-3692. 
Vorlicek TP, MD Kahn, Y Kasuza \& GR Helz. 2004. Capture of molybdenum in pyrite-forming sediments: role of ligandinduced reduction by polysulfides. Geochimica et Cosmochimica Acta 68: 547-556.

Wheat CG \& MJ Mottl. 2000. Composition of pore and spring waters from Baby Bare: Global implications of geochemical fluxes from a ridge flank hydrothermal system. Geochimica et Cosmochimica Acta 64: 629-642.
Williams G, AD Anbar \& GL Arnold. 2004. Reliability of black shales as recorders of marine molybdenum isotopes and paleoredox. AbSciCon 2004, NASA Ames Research Center, Moffett Field, CA. International Journal of Astrobiology, Supplement 1: 38-38.

Zheng Y, RF Anderson, A van Geen \& J Kuwabara. 2000. Authigenic molybdenum formation in marine sediments: a link to pore water sulfide in the Santa Barbara Basin. Geochimica et Cosmochimica Acta 64: 4165-4178.

Recibido el 18 de noviembre de 2008 y aceptado el 29 de mayo de 2009 